\title{
Hyperspectral imaging for monitoring oxygen saturation levels during normothermic kidney perfusion
}

\author{
Florian Tetschke ${ }^{1}$, Wenke Markgraf ${ }^{2}$, Marian Gransow $^{1}$, Susanne Koch $^{1}$, Christine Thiele $^{1}$, \\ Axel Kulcke ${ }^{3}$, and Hagen Malberg ${ }^{1}$ \\ ${ }^{1}$ Institut für Biomedizinische Technik, Dresden University of Technology, 01062 Dresden, Germany \\ ${ }^{2}$ UniversitätsCentrum für Orthopädie und Unfallchirurgie, Universitätsklinikum Carl-Gustav-Carus Dresden, \\ Dresden University of Technology, 01307 Dresden, Germany \\ ${ }^{3}$ Diaspective Vision GmbH, Strandstrasse 15, 18233 Pepelow, Germany \\ Correspondence to: Florian Tetschke (florian.tetschke@tu-dresden.de)
}

Received: 22 February 2016 - Revised: 3 June 2016 - Accepted: 23 July 2016 - Published: 22 August 2016

\begin{abstract}
The development of improved preservation techniques and the reliable assessment of donor grafts are main fields of research in transplantation medicine. Normothermic machine perfusion (NMP) is a promising alternative to static cold storage of organs, maintaining physiological conditions during preservation. In combination with NMP, we introduce hyperspectral imaging (HSI) as a novel approach for the monitoring of physiological kidney parameters. A line-scan HSI camera system was used to record images of porcine kidneys during NMP. Based on a dual-wavelength algorithm, the oxygen saturation levels were calculated from HSI recordings. Furthermore, we observed HSI images in the near-infrared (NIR) range in order to detect water characteristics of the kidney tissue. We found increasing levels of oxygenation during NMP and could discriminate between perfused and non-perfused areas. Cysts at the renal capsula were characterized by an absorption increase in the NIR band. Within this work, we showed that HSI is able to detect relevant chemical changes during NMP and allows the identification of pathologic variations.
\end{abstract}

The current situation in transplantation medicine is characterized by an acute lack of available donor grafts, particularly in the field of kidney transplantation. In order to expand the donor pool, an increasing number of marginal organs from donors with pre-existing risk factors (e.g., donors with hypertension or diabetes mellitus, age $>70$ years or death from cerebrovascular accident) are considered for transplantation (Reese et al., 2006). Accordingly, these organs are often associated with functional limitations (Querard et al., 2016; Pascual et al., 2008). Current research focuses on the improvement of preservation techniques as well as on the development of methods for the functional assessment of organs. As a promising alternative to static cold storage of the organ in an icebox, normothermic machine perfusion (NMP) allows the minimization of ischemia time and the obtainment of physiological kidney parameters prior to transplantation.
Studies about the advantageous effect of NMP on functional parameters of the kidney have aroused an increased interest in the field of NMP in recent years (Hosgood et al., 2015, 2011; Tillou et al., 2014). In combination with NMP, camerabased approaches such as hyperspectral imaging (HSI) allow space- and time-resolved imaging of clinical-relevant information and thus enable a more differentiated assessment of organ status (Best et al., 2013). Based on the unique feature to represent spatial and spectral information at the same time, HSI is a very suitable method for many medical applications and has been applied in a variety fields of research, e.g., for characterization of tumor tissue (Lu et al., 2014), for the analysis of wound healing (Calin et al., 2015) or in ophthalmology (Mordant et al., 2014). Depending on the application, a spectrum of light between 400 and $2500 \mathrm{~nm}$ is used to probe an object. In the range of $700-1300 \mathrm{~nm}$, also called bio-optical window, the light penetration in tissue has its maximum depth (Jobsis, 1977). Within this win- 
dow, local absorption maxima of the chromophores oxyhemoglobin $\left(\mathrm{HbO}_{2}\right)$, deoxyhemoglobin $(\mathrm{Hb})$ and water $\mathrm{H}_{2} \mathrm{O}$ can be quantified. For oxygenation measurements, characteristic bands of $\mathrm{HbO}_{2}$ and $\mathrm{Hb}$ spectra are analyzed, where $\mathrm{Hb}$ has its highest absorptions peaks at 435 and $555 \mathrm{~nm}$ (Prahl, 1999). $\mathrm{HbO}_{2}$ spectra show distinct peaks at 410,540 and $575 \mathrm{~nm}$. Isosbestic points, where the absorption of $\mathrm{Hb}$ and $\mathrm{HbO}_{2}$ intersect, can be found at 522, 569, and $586 \mathrm{~nm}$ (Beach et al., 2007). Water, which may have implications for identifying edema, has its maximum absorption peak in the wavelength range of the used silicon detector at around $970 \mathrm{~nm}$ (Jacquemoud and Ustin, 2003). Within this work, we introduce HSI as a contactless and fast approach for imagingrelevant physiological parameters during NMP of isolated kidneys. First results of oxygen saturation measurements using HSI are presented in this paper and characteristics of altered tissue are discussed.

\section{Methods}

\subsection{Experimental setup}

\section{Normothermic machine perfusion}

Porcine kidneys were retrieved from the slaughterhouse process and flushed with a histidine-tryptophanketoglutarate (HTK) solution. For transportation to our laboratory, the kidneys were stored at $\approx 4{ }^{\circ} \mathrm{C}$ in an ice box. Preparation was done by removing superficial fat and cannulating the artery, the vein and the ureter. After preparation, the kidneys were pressure-controlled perfused with autologous blood in a perfusion system developed in our group (see Fig. 1). Within this system, autologous, heparinized blood is driven by a roller pump (ISMATEC MCP Process, Novodirect GmbH, Kehl, Germany) within a $6.35 \mathrm{~mm}$ silicone tubing (Raumedic AG, Germany) through an oxygenator (hilite 2800, Medos Medizintechnik AG, Stollberg, Germany), where it is oxygenated and heated to $35-37^{\circ} \mathrm{C}$. The artery and vein are connected through our own custom-developed vessel connector. The arterial pressure is monitored through a pressure sensor (ATP300, Harvard Apparatus, Holliston, United States) at the arterial outlet of the oxygenator. The kidney is bedded in a custom storage box with an integrated heating mat. The venous backflow is then recycled in a venous hard-shell reservoir (hilite MVC 4030, Medos Medizintechnik AG, Stollberg, Germany). In addition to the arterial pressure, the system allows the monitoring and logging of data of the blood flow, the temperature of the perfusate and the heating unit, as well as the calculated resistance index, which is simply the quotient of the blood flow and arterial pressure. Perfusion parameters can be controlled and displayed by a touchscreen monitor with a custom-built user interface, based on Labview (National Instruments, Texas, Massachusetts, United States). Additionally, we integrated

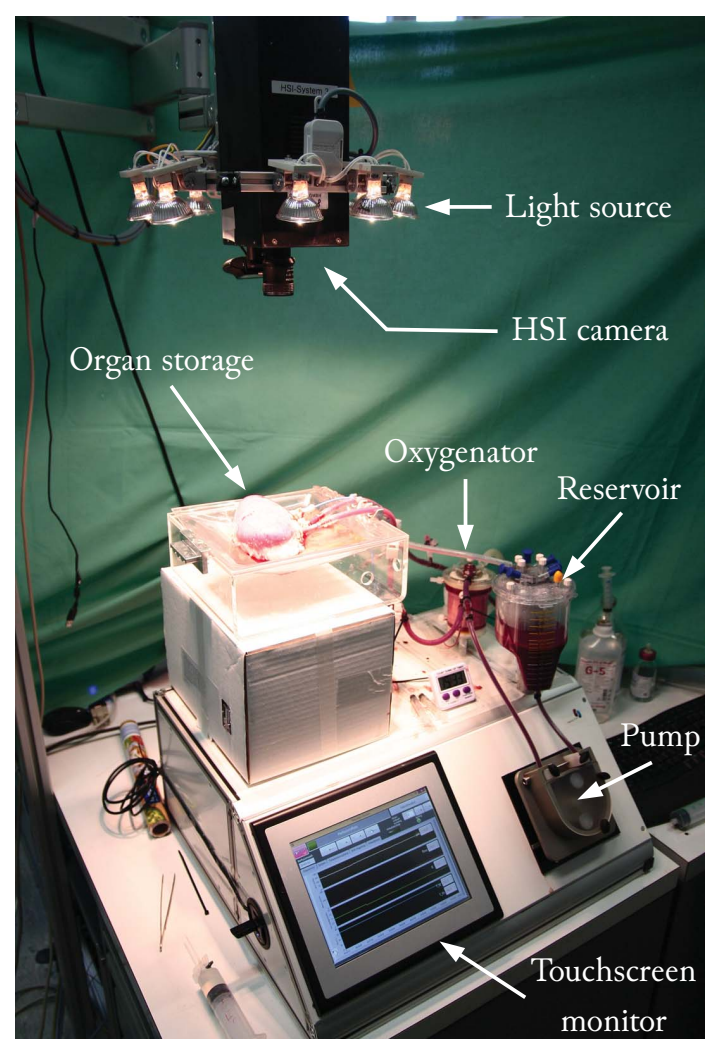

Figure 1. Measurement setup for hyperspectral imaging during normothermic kidney perfusion with the perfusion system developed at the Institute of biomedical engineering, Technical University Dresden.

several control algorithms for the automatic control of the arterial pressure, the temperature and the adjustment of blood gas parameters.

\section{Hyperspectral image instrumentation}

For acquisition of hyperspectral images, we used a linescan camera (TI-CAM, Diaspective Vision GmbH, Pepelow, Germany), based on the principle of a push-broom scanner (Lu and Fei, 2014). The CMOS image sensor (PhotonFocus AG, Lachen, Switzerland) is sensitive to a wavelength range of 400-1030 nm and offers a spatio-spectral resolution of $1312 \times 1082$ pixels. Each recorded line contains the information of one spatial axis and its corresponding spectrum $(x-$ $\lambda)$. The entire hypercube $(x-y-\lambda)$ is generated by moving the line with the internal entrance slit along the second spatial axis $(y)$ (see Fig. 2).

The camera was positioned at a distance of $35 \mathrm{~cm}$ to the kidney storage. For broadband illumination we used $8 \times 20$ W quartz-tungsten-halogen spots (OSRAM Decostar 51 ALU, Osram GmbH, Munich, Germany) with an aluminium reflector in order to provide sufficient radiation in the near-infrared (NIR) range. The spots were positioned sym- 


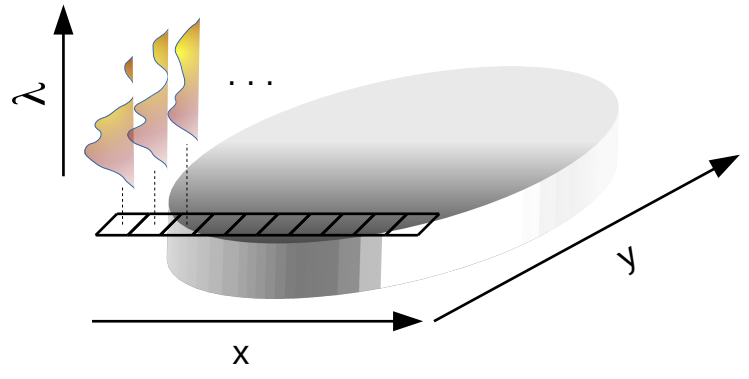

Figure 2. Schematic illustration of line-scan HSI acquisition. Each line records an $x-\lambda$ image. The entire hypercube is generated by moving the line along the $y$ axis.

metrically around the camera (see Fig. 1) and were powered by a $12 \mathrm{~V}$ DC custom-built power supply.

\subsection{Preprocessing and data analysis}

Hyperspectral images were recorded using the HSI acquisition software SICap GB (inno-spec GmbH, Nuremberg, Germany). For pre-processing and analysis we used custom routines written in MATLAB (The MathWorks, Natick, Massachusetts, United States). The algorithm for data analysis included the following steps:

\section{White and dark image acquisition}

For each experiment, white images $W_{x, y}(\lambda)$ of a standard reflectance surface (SphereOptics $\mathrm{GmbH}$, Oberuhldingen, Germany) with $99 \%$ diffuse reflectance were recorded. Additionally, a dark image $D_{x, y}$ was recorded in order to determine the dark current noise of the sensor (independent of $\lambda$ ). Therefore, the camera shutter was closed to prevent any light from penetrating into the camera.

\section{Raw image acquisition}

Raw intensity images $I_{x, y}(\lambda)$ of the kidneys were recorded during NMP, containing the uncorrected raw intensity of the scenery.

\section{White and dark image normalization}

In order to correct illumination nonuniformity and to remove variations caused by the dark current of the sensor, $I_{x, y}(\lambda)$ was normalized by $W_{x, y}(\lambda)$ and $D_{x, y}$, resulting in the reflectance $R_{x, y}(\lambda)$ :

$$
R_{x, y}(\lambda)=\frac{I_{x, y}(\lambda)-D_{x, y}}{W_{x, y}(\lambda)-D_{x, y}}
$$

\section{Absorbance calculation}

The absorbance $\operatorname{Abs}_{x, y}(\lambda)$ was calculated for each pixel using the modified Beer-Lambert law (Clark and Roush, 1984; Sassaroli and Fantini, 2004):
$\operatorname{Abs}_{x, y}(\lambda)=-\log _{10}\left[R_{x, y}(\lambda)\right]$.

\section{Noise filtering}

A Savitzky-Golay filter was applied to reduce noise in the absorbance spectra. This filter is based on a simplified least square fit convolution and already has proven to be valuable for HSI applications in earlier studies (King et al., 1999; Mahlein et al., 2012). The filter uses the number of adjacent values of the spectra (window length) and the order of the polynomial regression as input parameters (Savitzky and Golay, 1964). For smoothing, we used a window of 40 spectral values and a polynomial order of 3 .

\section{Spectral normalization}

In order to compare across a heterogeneous set, systematic variations between spectra were removed by normalization of the spectral absorbance intensity to the intensity between 568 and $570 \mathrm{~nm}$, where the absorption spectrum of hemoglobin shows an isosbestic point (Prahl, 1999). Therefore, the normalized absorbance $\operatorname{AbsNorm}_{x, y}(\lambda)$ was calculated by division of the absorbance at each wavelength $\operatorname{Abs}_{x, y}(\lambda)$ with the absorbance at $569 \mathrm{~nm} \mathrm{Abs}_{x, y}(569 \mathrm{~nm})$ :

$\operatorname{AbsNorm}_{x, y}(\lambda)=\frac{\operatorname{Abs}_{x, y}(\lambda)}{\operatorname{Abs}_{x, y}(569 \mathrm{~nm})}$.

\section{Oxygen saturation calculation}

Based on the normalized absorption spectrum of the kidney, oxygen saturation was calculated according to Beach et al. (1999). For this purpose, the ratio between the absorption of an oxygen-sensitive wavelength at $600 \mathrm{~nm}\left(\operatorname{AbsNorm}_{x, y}(600 \mathrm{~nm})\right)$ and an oxygen-insensitive wavelength at $569 \mathrm{~nm}\left(\mathrm{AbsNorm}_{x, y}\right.$ $(569 \mathrm{~nm})$ ) was determined. The absorbance ratio (AR) is inversely proportional to the oxygen saturation level within the kidney, denoted as $\mathrm{sO}_{2} \cdot \mathrm{sO}_{2}$ specifies the ratio of $\mathrm{HbO}_{2}$ and $\mathrm{Hb}$ in percent:

$$
\operatorname{AR}_{x, y}=\frac{\operatorname{AbsNorm}_{x, y}(600 \mathrm{~nm})}{\operatorname{AbsNorm}_{x, y}(569 \mathrm{~nm})} \approx \frac{1}{\mathrm{sO}_{2}} .
$$

\section{Application to calibration data}

For the acquisition of calibration data, we prepared a blood circuit consisting of a blood pump, a reservoir, a blood gas analyzer, a VIS/NIR-permeable optical cuvette and an oxygenator. Blood collected from healthy, slaughter house porcines were adjusted to physiological conditions $\left(\mathrm{pH}=7.35, T=37^{\circ} \mathrm{C}, \mathrm{Hkt}=30 \%\right)$. Reference blood probes with varying $\mathrm{sO}_{2}$ concentrations were produced by the adjustment of the $\mathrm{O}_{2}$ fraction of 


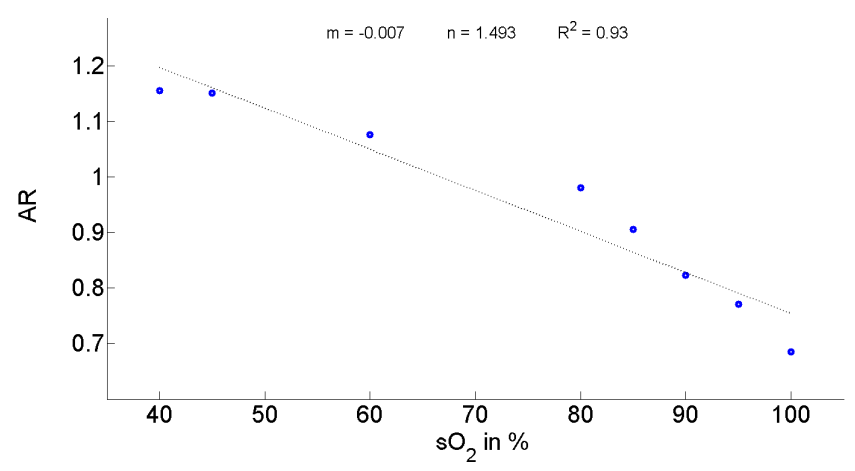

Figure 3. Correlation of calculated AR values from HSI measurements of calibration blood probes with the oxygen saturation of the blood probes (blue circles). The calculated oxygen saturation from HSI measurements during NMP is based on the linear regression of $\mathrm{AR}$ and oxygen saturation of the blood probes (dotted line).

the gas, which is delivered to the oxygenator by a gas mixer. Hereby, $\mathrm{sO}_{2}$ concentrations of $40,45,60,80$, $85,90,95$ and $100 \% \mathrm{sO}_{2}$ were obtained and monitored by an online blood gas monitor (CDI Blood Parameter Monitoring System 500, Terumo Corporation, Tokyo, Japan). The sensor of the system was integrated in a flow-through cuvette in the tubing line at the outlet of the oxygenator. For each $\mathrm{sO}_{2}$ saturation probe, we recorded HSI images and calculated $\mathrm{AR}_{x, y}$ from pixels belonging to the optical window of the cuvette. A simplified calibration model was built, based on the linear regression (Fig. 3, dotted line) of the calculated $\mathrm{AR}_{x, y}$ and the corresponding $\mathrm{sO}_{2}$. The resulting graph is represented in Fig. 3. The linear calibration model was applied to the $\mathrm{AR}_{x, y}$ values from the HSI measurements of the kidney. $\mathrm{sO}_{2}$ percentage is color-coded: red indicating high saturation, blue indicating low saturation.

\section{Results}

Mean acquisition time for an entire hypercube of the kidney was $10 \mathrm{~s}$. Random sample measurements with an infrared laser showed no significant increase of the temperature at the renal capsule, which possibly had been caused by the quartz-tungsten-halogen spots. Figure 4a shows the color-coded $\mathrm{sO}_{2}$ distribution of a HTK-flushed kidney before perfusion. The renal capsule is characterized by a relatively low mean oxygen saturation $\left(\mathrm{sO}_{2}\right.$, mean $\left.=37 \%\right)$. Higher oxygen levels $(>80 \%$ ) can be found around the vessels, where possibly blood from the surrounding tissue had been leaked. The renal capsule was perfused homogeneously and mean oxygen saturation significantly increased $\left(\mathrm{sO}_{2}\right.$, mean $\left.=98 \%\right)$ after a perfusion time of $120 \mathrm{~min}$ (see Fig. 4b). Glare artifacts appear as white spots at the surface of the kidney and in the storage box. Spectral properties (Fig. 4, left side) of the perfused kidney show characteristic absorption peaks of

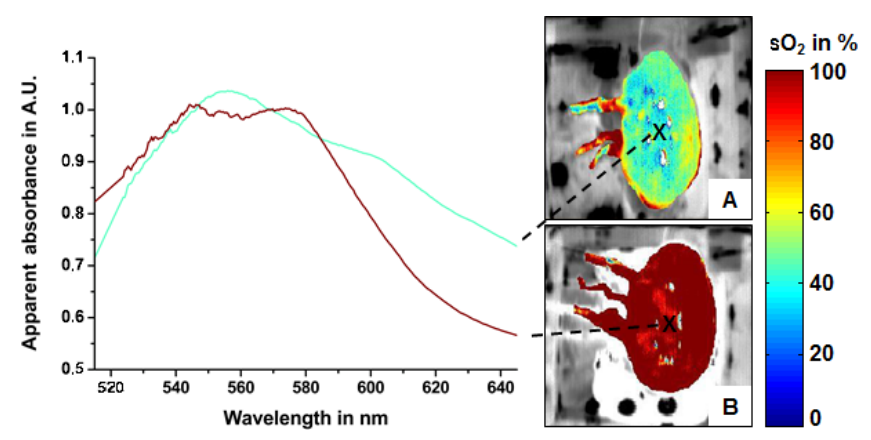

Figure 4. Hyperspectral images of a kidney with color-coded oxygen saturation distribution before (a) and after (b) $120 \mathrm{~min}$ of normothermic machine perfusion. The left side of the image shows the characteristic spectra of one pixel of the corresponding oxygenation status.

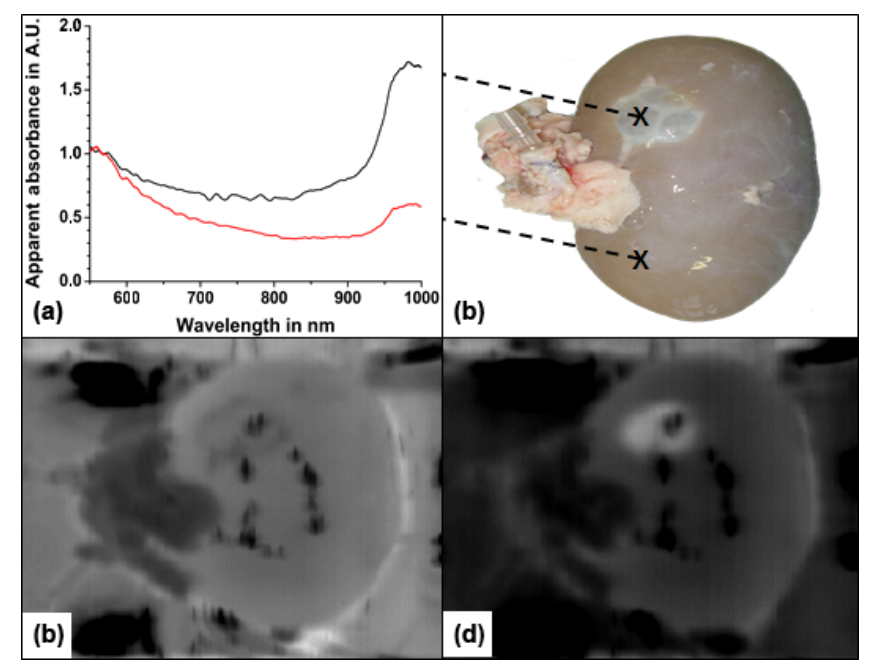

Figure 5. Kidney with a cyst, shown as RGB image (b), absorption image at $600 \mathrm{~nm}$ (c) and at $970 \mathrm{~nm}$ (d). The corresponding spectrum shows the spectral differences between the region with the cyst (black line) and tissue with no abnormalities (red line), (a).

oxygenated hemoglobin between $530 \mathrm{~nm}$ and $590 \mathrm{~nm}$ (red line), whereas these characteristics completely disappear in the recording of the HTK flushed kidney (blue line).

A RGB image of a kidney with a superficial cyst is shown in Fig. 5b. The cyst becomes considerably more pronounced in the absorption image at $970 \mathrm{~nm}$ (Fig. 5d), compared to the absorption image at $600 \mathrm{~nm}$ (Fig. 5c). Correspondingly, the spectrum of the cyst shows a significant absorption increase in the NIR wavelength range around $970 \mathrm{~nm}$ (Fig. 5a). 


\section{Discussion}

Within this work, we presented the application of a HSI system during NMP for the first time. In contrast to commonly used methods for the determination of the oxygenation status, HSI allows a pixel-wise analysis of chemical changes. The experimental results of HSI-based oxygen saturation calculation showed that HSI is suitable for the monitoring of the oxygen saturation distribution and the identification of areas with a reduced oxygen supply prior to transplantation. Previous works in this field evaluated tissue perfusion of in vivo perfused kidneys and proposed HSI for postoperative assessment (Olweny et al., 2013; Tracy et al., 2010; Holzer et al., 2011; Zuzak et al., 2011; Crane et al., 2008). In addition to the characterization of water-enriched tissue around $970 \mathrm{~nm}$, the analysis of lipid-sensitive regions in the spectral NIR band might be interesting for the correlation with ischemic injury (van Veen et al., 2004). Data analysis based on HSI is limited by the low penetration depth of the propagating light in the tissue. Broadband illumination ranging from 500 to $1000 \mathrm{~nm}$ has only a penetration depth of millimeters to a few centimeters, depending on the attenuation coefficient of the observed tissue. Hence, tissue injuries in deeper regions can not be detected. However, based on the fact that most of the metabolic activity occurs in the cortex of the kidney (Soltoff, 1986; Simmons et al., 2008), the application of HSI in combination with NMP of isolated kidneys is a favorable and easy-to-use method for the assessment of the organs status and for chemical imaging. It can be recognized in the images of the kidneys that the integration of a direct reflected light unit causes glare artifacts on the surface of the kidney. These artifacts can be reduced either by application of image processing algorithms (Shih et al., 2015) or by use of diffuse materials or polarization filters in the optical pathway. The development of new sensor generations could add further improvements to the instrumentation setup. Current research focuses on the development of new sensor architectures with enhanced quantum efficiency. In the context of real-time analysis of biological tissue, snapshot sensor technology is becoming an emerging field of interest (Kester et al., 2011), allowing one to capture hypercubes during a single integration time of the detector array. However, to date snapshot sensors have comparably low spectral and spatial resolution. The application of high-resolution image sensors in combination with a fast data acquisition and analysis could widen the range of HSI in medical applications.

\section{Conclusions}

In this work we presented a novel approach for the monitoring and visualization of relevant chemical changes of isolated kidneys prior to transplantation. We conclude that camerabased measurements such as HSI are easy to integrate in a perfusion setup and allow a fast and noninvasive measurement of tissue characteristics. However, assessment of bio- logical markers based on HSI is limited by the low penetration depth of the light. Furthermore, the integration of a new sensor generation such as snapshot HSI sensors could reduce acquisition time and even would allow real-time perfusion imaging. Based on the fact that ischemia plays a major role in graft injury, the monitoring of oxygen supply with HSI might provide a potential marker for the assessment and early identification of functional limitations, particularly for marginal organs. Future work of the authors will comprise the improvement on algorithms for the determination of oxygen saturation and the water content, as well as the spectral characterization of ischemic-injury-related tissue changes based on multivariate approaches. Furthermore, an enhanced HSI camera will be integrated and the setup will be refined regarding homogeneity of illumination and the reduction of glare artifacts.

\section{Data availability}

The data presented in this paper are available on request from the corresponding author.

Acknowledgements. The authors are grateful for the expert advice and stimulating discussions with Eike Brunner. Furthermore, we would like to thank Juliane Putz, Martin Janssen and Ulf Aschenbrenner for their advice on medical questions. We appreciate the work of our students Luise Olbricht, Hans Liebscher, Philipp Feistel, Sebastian Lehne and Friedrich Lusky, who contributed to the integration of the HSI system and assisted during NMP

Edited by: R. Morello

Reviewed by: four anonymous referees

\section{References}

Beach, J., Ning, J., and Khoobehi, B.: Oxygen saturation in optic nerve head structures by hyperspectral image analysis, Curr. Eye Res., 32, 161-170, 2007.

Beach, J. M., Schwenzer, K. J., Srinivas, S., Kim, D., and Tiedeman, J. S.: Oximetry of retinal vessels by dual-wavelength imaging: calibration and influence of pigmentation, J. Appl. Physiol., 86, 748-758, 1999.

Best, S. L., Thapa, A., Jackson, N., Olweny, E., Holzer, M., Park, S., Wehner, E., Zuzak, K., and Cadeddu, J. A.: Renal oxygenation measurement during partial nephrectomy using hyperspectral imaging may predict acute postoperative renal function, J. Endourol., 27, 1037-1040, 2013.

Calin, M. A., Coman, T., Parasca, S. V., Bercaru, N., Savastru, R., and Manea, D.: Hyperspectral imaging-based wound analysis using mixture-tuned matched filtering classification method, J. Biomed. Opt., 20, 046004, doi:10.1117/1.JBO.20.4.046004, 2015.

Clark, R. N. and Roush, T. L.: Reflectance spectroscopy - Quantitative analysis techniques for remote sensing applications, J. 
Geophys. Res., 89, 6329-6340, doi:10.1029/JB089iB07p06329, 1984.

Crane, N. J., Pinto, P. a., Hale, D., Gage, F. a., Tadaki, D., Kirk, A. D., Levin, I. W., and Elster, E. a.: Non-invasive monitoring of tissue oxygenation during laparoscopic donor nephrectomy, BMC surgery, 8, doi:10.1186/1471-2482-8-8, 2008.

Holzer, M. S., Best, S. L., Jackson, N., Thapa, A., Raj, G. V., Cadeddu, J. a., and Zuzak, K. J.: Assessment of renal oxygenation during partial nephrectomy using hyperspectral imaging, J. Urology, 186, 400-404, doi:10.1016/j.juro.2011.03.162, 2011.

Hosgood, S. a., Bagul, A., and Nicholson, M. L.: Minimising cold ischaemic injury in an experimental model of kidney transplantation., Eur. J. Clin. Invest., 41, 233-240, doi:10.1111/j.13652362.2010.02396.x, 2011.

Hosgood, S. a., Barlow, a. D., Hunter, J. P., and Nicholson, M. L.: Ex-vivo normothermic perfusion for quality assessment of marginal donor kidney transplants, Brit. J. Surg., 102, 14331440, doi:10.1002/bjs.9894, 2015.

Jacquemoud, S. and Ustin, S.: Application of radiative transfer models to moisture content estimation and burned land mapping, in: 4th International Workshop on Remote Sensing and GIS Applications to Forest Fire Management, 3-12, Ghent, Belgium, 57 June 2003.

Jobsis, F. F.: Noninvasive, infrared monitoring of cerebral and myocardial oxygen sufficiency and circulatory parameters, Science, 198, 1264-1267, 1977.

Kester, R. T., Bedard, N., Gao, L., and Tkaczyk, T. S.: Real-time snapshot hyperspectral imaging endoscope, J. Biomed. Opt., 16, 056005, doi:10.1117/1.3574756, 2011.

King, R. L., Ruffin, C., LaMastus, F. E., and Shaw, D. R.: The analysis of hyperspectral data using Savitzky-Golay filteringPractical issues (Part 2), Proc. IEEE IGARSS, 1, 398-400, doi:10.1109/IGARSS.1999.773512, 1999.

Lu, G. and Fei, B.: Medical hyperspectral imaging: a review, J. Biomed. Opt., 19, 10901, doi:10.1117/1.JBO.19.1.010901, 2014.

Lu, G., Halig, L., Wang, D., Chen, Z. G., and Fei, B.: Hyperspectral Imaging for Cancer Surgical Margin Delineation: Registration of Hyperspectral and Histological Images, Proc. SPIE Int. Soc. Opt. Eng., 9036, 90360S, doi:10.1117/12.2043805, 2014.

Mahlein, A. K., Steiner, U., Hillnhutter, C., Dehne, H. W., and Oerke, E. C.: Hyperspectral imaging for small-scale analysis of symptoms caused by different sugar beet diseases, Plant Methods, 8, 3, doi:10.1186/1746-4811-8-3, 2012.

Mordant, D. J., Al-Abboud, I., Muyo, G., Gorman, A., Harvey, A. R., and McNaught, A. I.: Oxygen saturation measurements of the retinal vasculature in treated asymmetrical primary openangle glaucoma using hyperspectral imaging, Eye (Lond), 28, 1190-1200, 2014.

Olweny, E. O., Faddegon, S., Best, S. L., Jackson, N., Wehner, E. F., Tan, Y. K., Zuzak, K. J., and Cadeddu, J. a.: Renal oxygenation during robot-assisted laparoscopic partial nephrectomy: characterization using laparoscopic digital light processing hyperspectral imaging., Journal of endourology / Endourological Society, 27, 265-269, doi:10.1089/end.2012.0207, 2013.
Pascual, J., Zamora, J., and Pirsch, J. D.: A systematic review of kidney transplantation from expanded criteria donors, Am. J. Kidney Dis., 52, 553-586, 2008.

Prahl, S.: Optical Absorption of Hemoglobin, available at: http: //omlc.org/spectra/hemoglobin/index.html (last access: 19 January 2016), 1999.

Querard, A. H., Foucher, Y., Combescure, C., Dantan, E., Larmet, D., Lorent, M., Pouteau, L. M., Giral, M., and Gillaizeau, F.: Comparison of survival outcomes between Expanded Criteria Donor and Standard Criteria Donor kidney transplant recipients: a systematic review and meta-analysis, Transpl. Int., 29, 403415, doi:10.1111/tri.12736, 2016.

Reese, P. P., Caplan, A. L., Kesselheim, A. S., and Bloom, R. D.: Creating a medical, ethical, and legal framework for complex living kidney donors, Clin. J. Am. Soc. Nephrol., 1, 1148-1153, 2006.

Sassaroli, A. and Fantini, S.: Comment on the modified BeerLambert law for scattering media, Phys. Med. Biol., 49, N255, doi:10.1088/0031-9155/49/14/N07, 2004.

Savitzky, A. and Golay, M. J. E.: Smoothing and Differentiation of Data by Simplified Least Squares Procedures, Anal. Chem., 36, 1627-1639, 1964.

Shih, Y., Krishnan, D., Durand, F., and Freeman, W. T.: Reflection removal using ghosting cues, in: IEEE Conference on Computer Vision and Pattern Recognition, CVPR2015, Boston, MA, USA, 7-12 June 2015, 3193-3201, doi:10.1109/CVPR.2015.7298939, 2015.

Simmons, M. N., Schreiber, M. J., and Gill, I. S.: Surgical renal ischemia: a contemporary overview, J. Urol., 180, 19-30, 2008.

Soltoff, S. P.: ATP and the regulation of renal cell function, Annu. Rev. Physiol., 48, 9-31, 1986.

Tillou, X., Thuret, R., and Doerfler, A.: Ischemia/reperfusion during normothermic perfusion., Progrès en urologie : journal de l'Association française d'urologie et de la Société française d'urologie, 24 Suppl 1, S51-5, doi:10.1016/S11667087(14)70064-8, 2014.

Tracy, C. R., Terrell, J. D., Francis, R. P., Wehner, E. F., Smith, J., Litorja, M., Hawkins, D. L., Pearle, M. S., Cadeddu, J. a., and Zuzak, K. J.: Characterization of renal ischemia using DLP hyperspectral imaging: a pilot study comparing artery-only occlusion versus artery and vein occlusion., J. Endourol., 24, 321-325, doi:10.1089/end.2009.0184, 2010.

van Veen, R. L., Sterenborg, H., Pifferi, A., Torricelli, A., and Cubeddu, R.: Determination of VIS- NIR absorption coefficients of mammalian fat, with time- and spatially resolved diffuse reflectance and transmission spectroscopy, in: Biomedical Topical Meeting, p. SF4, Optical Society of America, doi:10.1364/BIO.2004.SF4, 2004.

Zuzak, K. J., Francis, R. P., Wehner, E. F., Litorja, M., Cadeddu, J. a., and Livingston, E. H.: Active DLP hyperspectral illumination: a noninvasive, in vivo, system characterization visualizing tissue oxygenation at near video rates, Anal. Chem., 83, 74247430, doi:10.1021/ac201467v, 2011. 